\title{
Epidemiological and genomic characterization of community-acquired Clostridium difficile infections
}

\author{
Christina S. Thornton 1,2, Joseph E. Rubin ${ }^{3,4}$, Alexander L. Greninger ${ }^{5,7}$, Gisele Peirano ${ }^{3}$, Charles Y. Chiu ${ }^{5,8}$ \\ and Dylan R. Pillai ${ }^{1,2,3,6,9^{*}}$ (D)
}

\begin{abstract}
Background: Clostridium difficile infection (CDI) is a major cause of morbidity and mortality in North America and Europe. The aim of this study was to identify epidemiologically-confirmed cases of community-acquired (CA)-CDI in a large North American urban center and analyze isolates using multiple genetic and phenotypic methods.

Methods: Seventy-eight patients testing positive for C. difficile from outpatient clinics were further investigated by telephone questionnaire. CA-CDI isolates were characterized by antibiotic susceptibility, pulsed-field gel electrophoresis and whole genome sequencing. CA-CDI was defined as testing positive greater than 12 weeks following discharge or no previous hospital admission in conjunction with positive toxin stool testing.

Results: $51.3 \%$ (40/78) of the patients in this study were found to have bona fide CA-CDI. The majority of patients were female $(71.8 \%$ vs. $28.2 \%)$ with $50-59$ years of age being most common (21.8\%). Common co-morbidities included ulcerative colitis (1/40; $2.5 \%)$, Crohn's disease (3/40; 7.5\%), celiac disease (2/40; 5.0\%) and irritable bowel syndrome (8/40; 20.0\%). However, of 40 patients with CA-CDI, 9 (29.0\%) had been hospitalized between 3 and 6 months prior and 31 (77. 5\%) between 6 and 12 months prior. The hypervirulent North American Pulostype (NAP) 1-like (9/40; 22.5\%) strain was the most commonly identified pulsotype. Whole genome sequencing of CA-CDI isolates confirmed that NAP 1-like pulsotypes are commonplace in CA-CDI. From a therapeutic perspective, there was universal susceptibility to metronidazole and vancomycin.
\end{abstract}

Conclusions: All CA-CDI cases had some history of hospitalization if the definition were modified to health care facility exposure in the last 12 months and is supported by the genomic analysis. This raises the possibility that even CA-CDI may have nosocomial origins.

\section{Background}

Clostridium difficile infections (CDI) are the most common cause of infectious diarrheal infection amongst hospitalized patients in North America and Europe [1]. CDI has recently surpassed methicillin-resistant Staphylococcus aureus as a hospital acquired infection [2, 3]. The incidence, mortality and associated health care costs associated with CDI are significant, with $10-25 \%$ of all cases of antibiotic-associated diarrheal onset attributed to CDI [4]. Clinically, nosocomial CDI has been well-studied with

\footnotetext{
* Correspondence: drpillai@ucalgary.ca

${ }^{1}$ Department of Microbiology and Infectious Diseases, University of Calgary, Calgary, AB, Canada

2Department of Medicine, University of Calgary, Calgary, AB, Canada

Full list of author information is available at the end of the article
}

several risk factors for acquisition including hospitalization at time of infection, prior hospitalization, older age $(>65)$, antibiotic therapy (in particular fluoroquinolones, cephalosporins and clindamycin $[5,6])$, use of nasogastric tubes, surgical procedures in the gastrointestinal tract, history of inflammatory bowel disease (IBD) and other states of immunosuppression [6,7]. The pathogenicity of CDI is primarily attributed to the production of two exotoxins, toxin $\mathrm{A}$ and $\mathrm{B}$ that cause subsequent clinical manifestation including colonic dysregulation and cellular death [8]. A "binary" toxin has also been reported that may further contribute to pathogenesis in certain strains [1]. Additionally, $\mathrm{CDI}$ is spore-forming, allowing survival in the physical environment for prolonged periods of time [9], which challenges infection control and prevention in the hospital

(c) The Author(s). 2018 Open Access This article is distributed under the terms of the Creative Commons Attribution 4.0 International License (http://creativecommons.org/licenses/by/4.0/), which permits unrestricted use, distribution, and 
setting. Several other factors including gut microbiota disruption by antibiotic use and host endogenous antibodies to the toxins have also been implicated in CDI disease progression $[6,7,10]$.

Recently, several studies have described the onset of community-acquired CDI (CA-CDI) [6]. The definition of CA-CDI requires the patient to not have been in a hospital or health care facility within the previous 12 weeks or to develop CDI symptoms within $48 \mathrm{~h}$ of hospital admission [11]. In contrast, nosocomial CDI requires that symptoms occur greater than $48 \mathrm{~h}$ after hospital admission or in less than 4 weeks after discharge from a health care facility [11]. Finally, indeterminate cases occur in the community between 4 and 12 weeks after hospital discharge has occurred [11]. CA-CDI rates are on the rise, with $20-45 \%$ [1,9] of all CDI cases attributed to community onset, and a further $22 \%$ of patients having no history of antimicrobial several months prior to CDI onset [12].

CA-CDI rates amongst the pediatric population have also increased over time [13]. The clinical significance of C. difficile detection in infants and young children less than two years old is unclear, as they have been established as asymptomatic carriers [14]. Nevertheless, children may still serve as a means of transmission to other individuals in the household by exposure to spores within the same physical environment [15]. Exposure to C. difficile in the outpatient setting may serve as a means of transmission [16]. Two-thirds of CA-CDI patients had some form of occupational exposure within health care fields that may have preceded their illness [17]. There is also evidence that food-borne exposure to $C$. difficile may be a means of transmission within the community as spores have been demonstrated to survive normal cooking temperatures [18]. Zoonotic reservoirs exist in several animals including cattle and pigs $[15,19-23]$.

The aim of this study was to better understand the impact of CA-CDI by identifying risk factors linked to epidemiologically confirmed CA-CDI cases in the metropolitan center of Calgary (approximately 1.3 million people). $C$. difficile isolates from CA-CDI cases were further characterized by antibiotic susceptibility testing, pulsed field gel electrophoresis and whole genome sequencing.

\section{Materials and methods}

\section{Study population}

Patients whose stool specimens were submitted from patient service centers to Calgary Laboratory Services and positive for $C$. difficile toxin between March and October 2012 were included in the study. These patients were included based on physician suspicion of $C$. difficile infection and test submission. The physician decision to test was not scrutinized by the study team but taken at face value. A control group of nosocomial strains were collected from inpatients admitted during the study period. All stools were diarrheal. A nosocomial strain was defined as a stool specimen positive for $C$. difficile from a patient admitted to one of the four major hospitals in the city of Calgary. Medical records including hospitalizations, relevant laboratory tests and diagnosis was obtained through a centralized provincial database. Ethical approval was obtained through University of Calgary Conjoint Health Research Ethics Board (ID 13-0406).

\section{Telephone survey}

For the purposes of this study, CA-CDI was defined as infection occurring greater than 12 weeks following discharge or no previous hospital admission. CA-CDI patients $(n=78)$ were, within 12 months of testing positive, assessed by telephone questionnaire for epidemiological data related to the mode of acquisition. The telephone study was designed to gauge risk factors traditionally associated with CDI acquisition reported in the literature. For patients who could not recall their last hospitalization, an electronic medical record (EMR) was interrogated to determine the date and site of visit if any.

\section{Specimen collection, testing, culture, and deoxyribonucleic acid (DNA) isolation}

Patients' stools were tested for CDI using a two-step testing algorithm which includes GDH screening by ELISA (Diasorin LIAISON ${ }^{\circ}$ C. difficile GDH) followed by toxin testing (Cepheid $\mathrm{Xpert}^{\circ} \mathrm{C}$. difficile/Epi) from outpatient physician offices. C. difficile isolates were selected on cycloserine cefoxitin fructose agar (CCFA) and species confirmed by matrix assisted laser desorption ionization-time of flight mass spectrometry (MALDI-TOF MS) Genomic DNA was extracted for whole genome sequencing using an in-house modified protocol from the QIAamp DNA Mini Extraction Kit (Qiagen, USA). Colonies were re-suspended in $0.9 \%$ saline to a McFarland standard of $2.4-3.0$ and subsequently centrifuged at $13,000 \mathrm{rpm}(\mathrm{rpm})$ on a tabletop centrifuge for one minute with supernatant discarded. The remaining pellet was re-suspended in $180 \mu \mathrm{L}$ of enzyme solution ( $20 \mathrm{mg} / \mathrm{mL}$ lysozyme, $20 \mathrm{mM}$ Tris- $\mathrm{HCl}, 2 \mathrm{mM}$ EDTA and 1.2\% Triton X). The solution was then incubated for $30 \mathrm{~min}$ at $37{ }^{\circ} \mathrm{C}$ until clearing of the solution was noted. Twenty $\mu \mathrm{L}$ of reconstituted Proteinase K solution (Sigma-Aldrich, USA) was added with a further incubation at $56{ }^{\circ} \mathrm{C}$ overnight. The remainder of the protocol followed instructions as denoted in the manufacturer's protocol. The final pellet was re-suspended in $50 \mu \mathrm{l}$ DNase/RNase free water.

\section{Antibiotic susceptibility testing}

In brief, cultured isolates grown on agar plates for $48 \mathrm{~h}$ were suspended in $0.9 \%$ saline and standardized to a 1.0 
McFarland density. The inoculum was then evenly spread over blood agar and incubated in anaerobic incubation conditions for $24 \mathrm{~h}$. Metronidazole and vancomycin E-test strips (BioMerieux, USA) were stored at $-20{ }^{\circ} \mathrm{C}$ until use. Following inoculation of plates, E-test strips were then placed and incubated for a further $48 \mathrm{~h}$ before measurement. Epidemiological cut-off (ECOFF) guidelines were used from EUCAST with the following breakpoint: vancomycin $>2 \mathrm{mg} / \mathrm{L}$ as non-susceptible and $<2 \mathrm{mg} / \mathrm{L}$ as susceptible; metronidazole $>2 \mathrm{mg} / \mathrm{L}$ as non-susceptible and $<2 \mathrm{mg} / \mathrm{L}$ as susceptible [24]. E-test strips were used to correlate with disk diffusion based on prior research demonstrating adequate agreement [25].

\section{Pulsed-field gel electrophoresis (PFGE)}

PFGE was conducted on CA-CDI isolates as outlined in previous methods [26, 27]. Briefly, DNA was prepared by lysis of cells within agarose plugs. The resultant plugs were then digested with SmaI as described previously [28]. A BioRad Chef Mapper was used to visualize the resulting fragments [27]. PFGE profiles were then compared using BioNumerics, version 5.1 (Applied Maths, Belgium) with standardized $C$. difficile NAP serotypes that were run as controls in parallel with samples. The resulting dendograms were then categorized using an unweighted-pair group method using average linkages (UPGMA), Dice similarity coefficient (optimization $1.5 \%$, tolerance $1 \%)[28,29]$. Clustering of PFGE results was performed using Advanced Cluster Analysis algorithm from BioNumerics, version 5.1 (Applied Maths, Belgium).

\section{Whole genome sequencing}

Next-generation sequencing libraries were prepared using quarter-volume reactions of Nextera XT (Illumina) and 1 nanogram of genomic DNA with 14 cycles of amplification. Barcoded libraries were pooled and sequenced on an Illumina HiSeq 2x250bp (nosocomial isolates; control group) and the Illumina MiSeq (CA-CDI isolates; study group). Two different sequencers were used due to logistical reasons. Only a subset of CA-CDI, indeterminate, and nosocomial isolates $(n=71)$ were sequenced due to study budgetary constraints. Paired-end reads were run through the nullarbor (https://github.com/tseemann/nullarbor) pipeline using the $C$. difficile 630 genome (NC_009089) as a reference. Phylogenetic trees were constructed based on the core genome SNP alignment using MrBayes (http://mrbayes.sourceforge.net/). The dendogram was viewed and exported using FigTree (http://tree.bio.ed.ac.uk/software/figtree/).

\section{Results}

Patients $(n=78)$ testing positive for CDI from outpatient physician offices were contacted by telephone questionnaire to assess epidemiological risk and exposures in detail. Further characterization based on clinical timelines reclassified patients into CA-CDI, defined as greater than 12 weeks following discharge or no previous hospital admission (40/78; 51.2\%; Table 1$)$. Overall, the majority of patients were female $(71.8 \%$ vs. $28.2 \%)$ and between 50 and 59 years of age $(21.8 \%)$.

The most common co-morbidities in CA-CDI patients included ulcerative colitis $(1 / 40 ; 2.5 \%)$, Crohn's Disease $(3 / 40 ; 7.5 \%)$, celiac disease $(2 / 40 ; 5.0 \%)$ and irritable bowel syndrome $(8 / 40 ; 20.0 \%)$ (Table 2$)$. Among the 40 patients with CA-CDI, 31 (77.5\%) had no recent hospitalizations within the last 6 months prior to infection. Twenty-one patients (52.5\%) had a household contact admitted to the hospital the year prior to onset of CDI. Seventeen patients $(42.5 \%)$ had a household contact that had confirmed CDI in the year prior.

PFGE analysis was done on CA-CDI isolates collected during the study. Figure 1 highlights the CA-CDI PFGE pulsotypes. Amongst the CA-CDI isolates, both NAP1 $(9 / 40 ; 22.5 \%)$ and NAP $4(8 / 40 ; 20 \%)$ were the most abundant, followed by NAP $2 / 11$ (5/40; 12.5\%), NAP 12 $(4 / 40 ; 10 \%)$, and NAP $6(4 / 40 ; 10.0 \%)$ (Table 3). Antibiotic susceptibility for metronidazole and vancomycin was tested for CA-CDI isolates received from the community. There was universal susceptibility amongst all isolates to metronidazole and vancomycin based on accepted interpretive breakpoints (data not shown).

Whole genome sequencing (WGS) was performed on a subset of epidemiologically confirmed CA-CDI isolates $(n=30)$. Figure 2 demonstrates the phylogenetic tree derived from whole genome sequencing of CA-CDI

Table 1 Demographics of Patients Contacted for Community C. difficile Study

\begin{tabular}{ll}
\hline Demographic Characteristics & CA-CDI Strains $^{a}$ \\
\hline Total & $40(51.2)$ \\
Gender & $11(27.5)$ \\
Male & $29(72.5)$ \\
Female & \\
Age & $1(2.5)$ \\
$<10$ & $1(2.5)$ \\
$10-19$ & $6(15.0)$ \\
$20-29$ & $2(5.0)$ \\
$30-39$ & $1(2.5)$ \\
$40-49$ & $8(20.0)$ \\
$50-59$ & $10(25.0)$ \\
$60-69$ & $5(12.5)$ \\
$70-79$ & $6(15.0)$ \\
$>80$ &
\end{tabular}

Defined as $>12$ weeks following discharge or no previous hospital admission. CA-CDI; community-acquired $C$. difficile infection 
Table 2 Telephone Survey Results of Patients Contacted for Community C. difficile Study

\begin{tabular}{|c|c|}
\hline & CA-CDI (\%) $)^{\mathrm{a}}$ \\
\hline Total & $40(51.2)$ \\
\hline Prior diagnosis of $\mathrm{CDI}$ & $15(37.5)$ \\
\hline \multicolumn{2}{|l|}{ Most recent hospitalization: } \\
\hline$>6$ months & $31(77.5)$ \\
\hline Within 6 months & $9(22.5)$ \\
\hline Within 3 months & $0(0.0)$ \\
\hline Previous week & $0(0.0)$ \\
\hline \multicolumn{2}{|l|}{ Times hospitalized in last 6 months: } \\
\hline 1 & $0(0.0)$ \\
\hline $2-5$ & $7(17.5)$ \\
\hline $5-10$ & $2(5.0)$ \\
\hline Prior history of Ulcerative Colitis & $1(2.5)$ \\
\hline Prior history of Crohn's Disease & $3(7.5)$ \\
\hline Prior history of Celiac disease & $2(5.0)$ \\
\hline Prior history of IBS & $8(20.0)$ \\
\hline Institutional care in prior year & $5(12.5)$ \\
\hline Visit someone in nursing home or hospital prior year before CDI & $17(42.5)$ \\
\hline Household contact in hospital in prior year before CDI infection & $21(52.5)$ \\
\hline Occupation in health care field & $4(44.4)$ \\
\hline Work with patients diagnosed with CDI in prior year & $1(2.5)$ \\
\hline Household contact hospitalized in year prior to CDI infection & $17(42.5)$ \\
\hline Household contact with CDI infection in year prior to CDI & $9(22.5)$ \\
\hline \multicolumn{2}{|l|}{ Children in household } \\
\hline No & $15(37.5)$ \\
\hline $0-1$ year & $8(20.0)$ \\
\hline $1-2$ years & $3(7.5)$ \\
\hline $2-5$ years & $10(25.0)$ \\
\hline $7-10$ years & $20(50.0)$ \\
\hline \multicolumn{2}{|l|}{ Household pets: } \\
\hline No & $16(40.0)$ \\
\hline Dogs & $9(22.5)$ \\
\hline Cats & $4(10.0)$ \\
\hline Fish & $2(5.0)$ \\
\hline Birds & $5(12.5)$ \\
\hline Other & $5(12.5)$ \\
\hline Occupational work related to pork products & $5(12.5)$ \\
\hline \multicolumn{2}{|l|}{ Consume pork products } \\
\hline No & $12(30.0)$ \\
\hline Yes, 1 time per week & $7(17.5)$ \\
\hline Yes, 2-4 times per week & $11(27.5)$ \\
\hline Yes, $>4$ times per week & $10(25.0)$ \\
\hline Vegetarian & $8(20.0)$ \\
\hline
\end{tabular}

${ }^{\mathrm{a} D e f i n e d}$ as $>12$ weeks following discharge or no previous hospital admission. CA-CDI; community-acquired C. difficile infection 


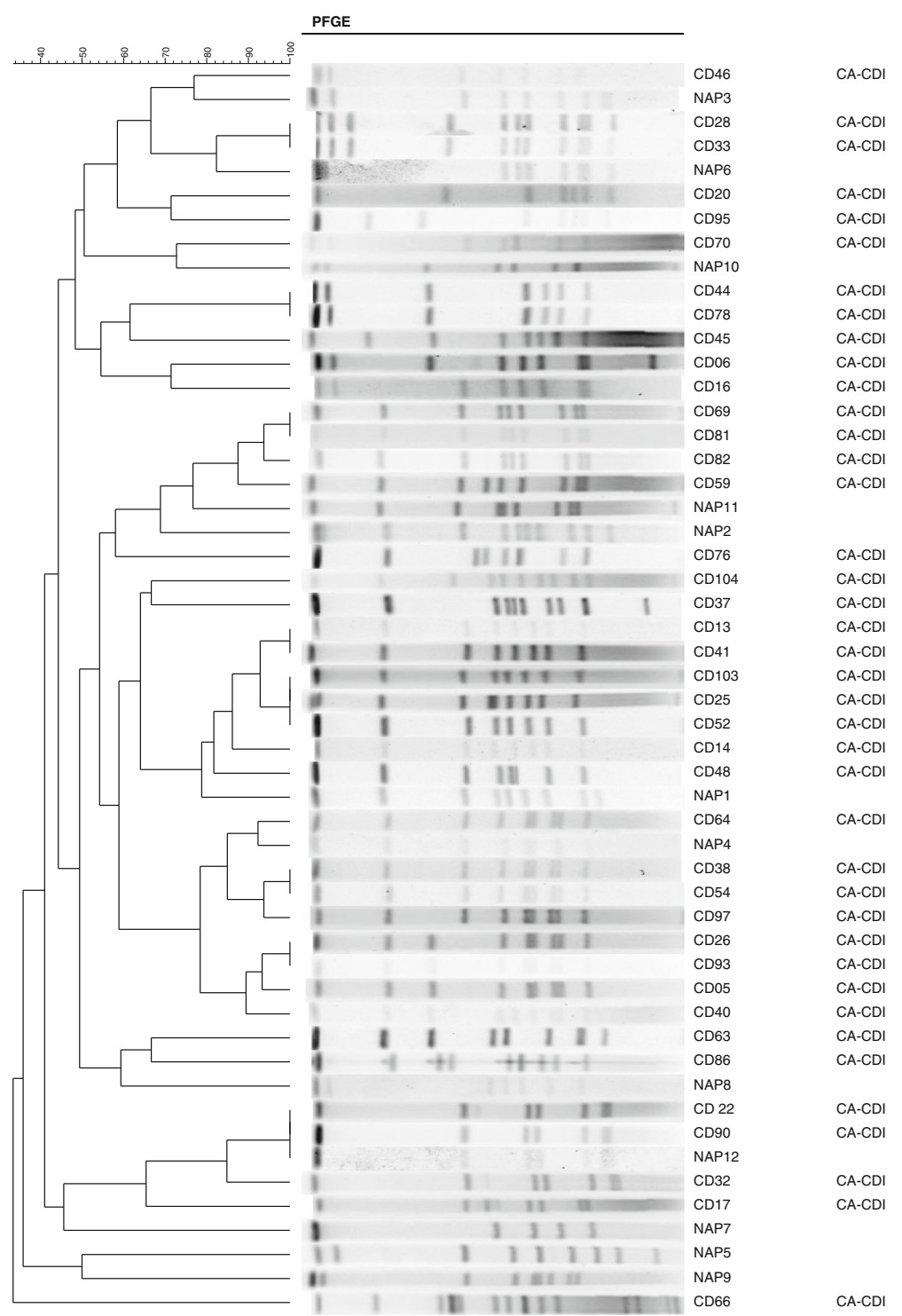

Fig. 1 Pulsed field gel electrophoresis from epidemiologically confirmed community- acquired C. difficile isolates (CA-CDI labelled "CD") compared to reference North American pulsotypes (labelled "NAP")

isolates. Concordance between PFGE and WGS was observed based on phylogenetic analysis in terms of clustering with reference North American pulsotypes. A NAP-1 like cluster (CD25, CD41, CD13, CD63, CD104, CD103, CD48 and CD52) was noted from the WGS phylogenetic tree (bottom of Fig. 2). This cluster isolates represented patients with no hospitalizations in the six months prior to positive stool toxin, suggesting the possibility of a community-acquired NAP-1 like cluster. There was no other significant epidemiological association noted between these patients.

In order to determine whether unique clusters exist in CA-CDI $(n=30)$ as compared to indeterminate isolates $(n=8)$ from this study or a set of nosocomial isolates $(n$
=33) collected from the hospitals during the study period, a WGS phylogenetic analysis was performed. Nosocomial isolates were obtained from a separate control group of patients admitted to hospital during the study period. A phylogenetic tree comparing all three epidemiological categories is presented in Fig. 3, demonstrating that CA-CDI and nosocomial strains are genomically indistinguishable.

\section{Discussion}

CA-CDI is associated with significant clinical complications. It is estimated that $40 \%$ of patients require hospitalization, $20 \%$ suffer severe infection, $4.4 \%$ go on to have severe complications, $20 \%$ end up with treatment 
Table 3 Community acquired (CA) C. difficile Isolates $(n=40)$ categorized by related North American Pulsotype (NAP)

\begin{tabular}{ll}
\hline NAP Type & CA-CDI (\%) \\
\hline NAP1 & $9(22.5)$ \\
NAP2/11 & $5(12.5)$ \\
NAP3 & $1(2.5)$ \\
NAP4 & $8(20.0)$ \\
NAP5 & $0(0.0)$ \\
NAP6 & $4(10.0)$ \\
NAP7 & $0(0.0)$ \\
NAP8 & $0(0.0)$ \\
NAP9 & $0(0.0)$ \\
NAP10 & $0(0.0)$ \\
NAP12 & $410.0)$ \\
Non-NAP & $9(22.5)$ \\
\hline
\end{tabular}

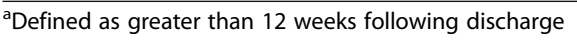

failure, and $28 \%$ have recurrent CDI [30]. An economic analysis from Canada found nearly 38,000 cases of CDI in 2012 with a total cost to society of $\$ 281$ million, in part due to in-hospital investigations, community management and productivity loss [31]. Furthermore, management of CDI relapses alone was over $\$ 65$ million. This has tremendous impact on the health care system in terms of costs, patient outcomes and burden on already limited resources [15]. We undertook to closely examine CA-CDI using a patient-based telephone questionnaire, to better understand this epidemiological group. We also analyzed the isolates using multiple laboratory methods to determine if unique traits associated with CA-CDI such as antibiotic resistance or genetic features exist.

Several studies have assessed epidemiology and risk factors associated with CA-CDI. One recent study from Japan [32] found the average age to be 58.8 with $50 \%$ > 65 years of age, similar to our findings. The gender distribution was also noted to be similar between studies (26.9\% male). Interestingly, this study found the majority of patients were more likely to have received antimicrobials (oral fluoroquinolones most notably) and antacids. A recent report from the US found $40 \%$ of patients with CA-CDI were not previously exposed to antimicrobials [33]. Our study did assess antimicrobial prescription through the telephone survey but resulted in a large proportion of patients unable to recall the timeframe of antibiotic use. The most striking epidemiological finding in the current study is that all CA-CDI cases had some history of hospitalization if the definition were modified to hospital exposure in the last 12 months rather than the last 12 weeks as currently accepted. This raises the possibility that even CA-CDI has nosocomial origins albeit remote.

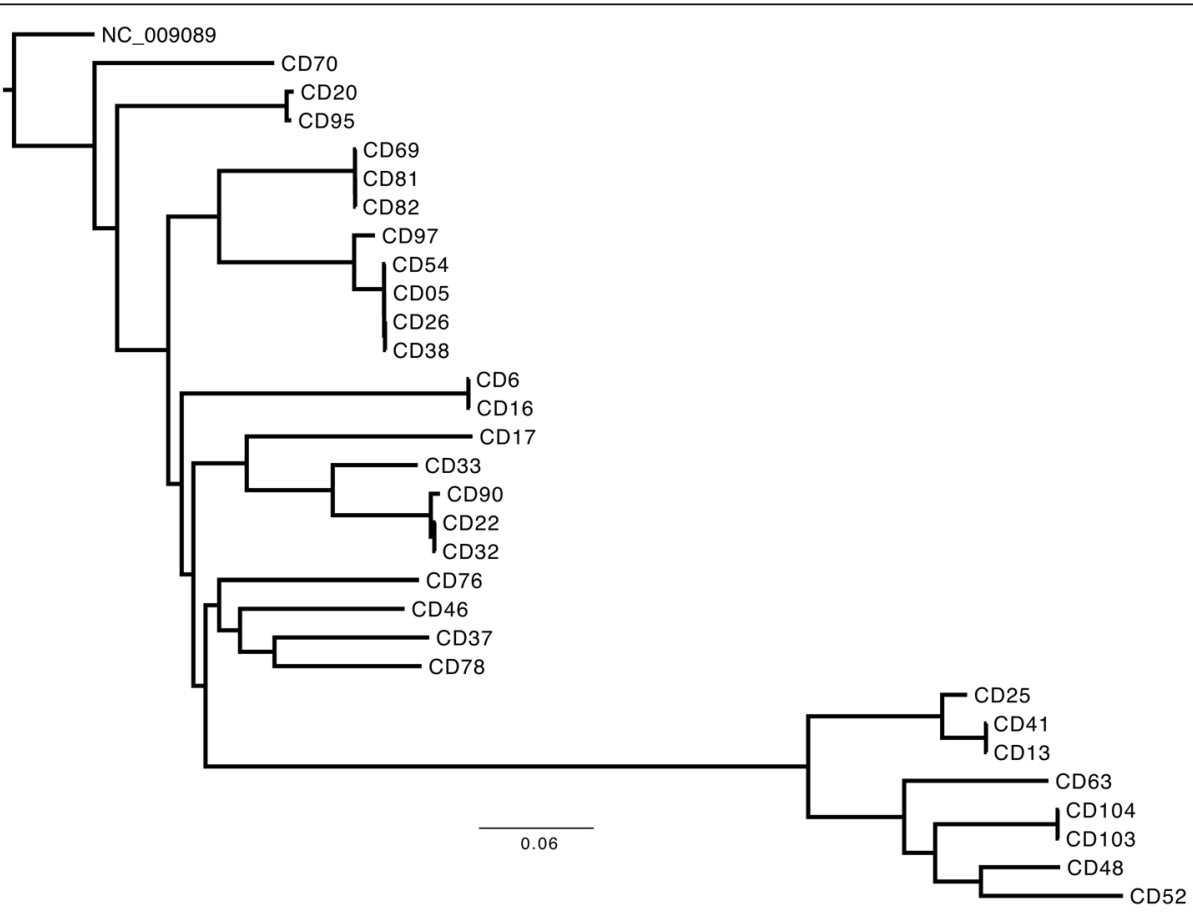

Fig. 2 Core genome SNP phylogeny of community-acquired isolates ("CD") sequenced in this study. Reads from 30 community-acquired C. difficile isolates (CA-CDI) were run through the nullarbor pipeline using C. difficile 630 genome (NC_009089) as the reference and core SNP genome phylogenies were constructed using MrBayes using the 630 reference genome as the outgroup. A NAP-1 like cluster (CD25..CD52) is noted. The bootstrap support values for all branches are 100 


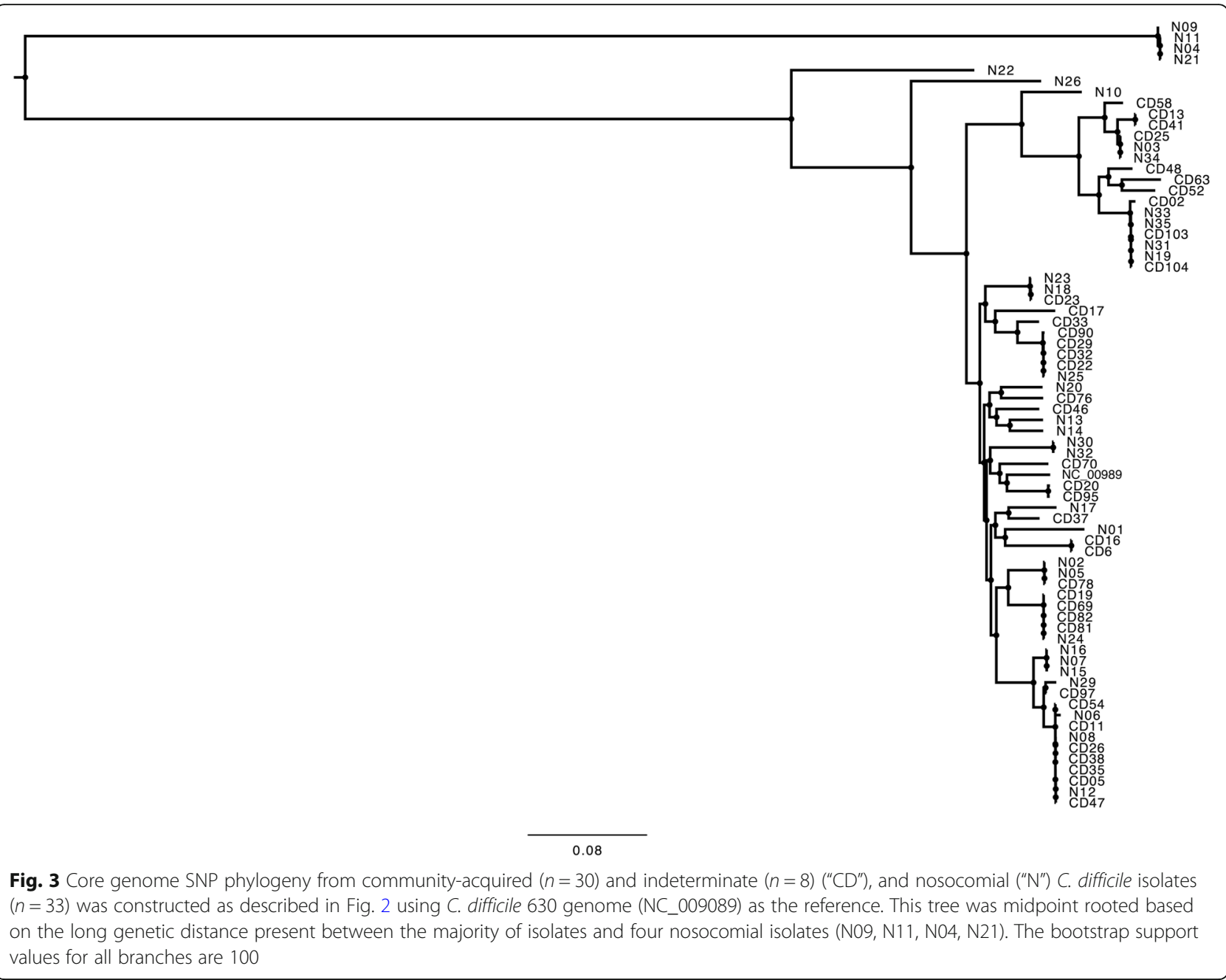

Metronidazole and vancomycin are considered first line agents in the clinical treatment of nosocomial $C$. difficile infection. The susceptibility observed with both antibiotics in our study suggests antibiotic resistance in CA-CDI is not a predisposing factor. There have been some reports [34] and in vitro data [35] suggestive of antibiotic resistance in nosocomial CDI, but limited data exists for CA-CDI. Furthermore, increasing resistance has been reported in toxigenic $C$. difficile strains. A study from Spain found resistance to metronidazole and intermediate resistance to vancomycin amongst nosocomial CDI, albeit with none resulting in treatment failure [36]. Ultimately, as antibiotic exposure increases in the CA-CDI population, it will be necessary to further monitor resistance patterns and assess clinical utility of standard antibiotic treatment regimens.

Molecular characterization by PFGE analysis and WGS demonstrated the predominance of the NAP1 ribotype in CA-CDI. The NAP1 hypervirulent strain has been associated with increased toxin production within animal models and increased antibiotic resistance, in particular to fluoroquinolones [37]. Reports exist of increased detrimental clinical outcomes including intensive care admission, requirement for colectomy, or death with this strain [38, 39]. Clinical reports of the hypervirulent NAP1 strain have been primarily associated thus far with nosocomial $C$. difficile infection. The presence of NAP 1 in the community with no recent hospital exposure implies that this strain can enter the nosocomial setting upon admission. Our WGS analysis suggests that CA-CDI isolates are indistinguishable from nosocomial isolates and no unique cluster is especially adapted to the community. CA-CDI essentially mirrors nosocomial isolates at the genomic level in this study. Microbiome analysis has been well elucidated in nosocomial infections, with significant dysbiosis shown in patients following infection [40-42]. Dysbiosis may also be a predisposing factor in the acquisition of CDI not only in the hospital but also in the community.

Limitations of this study include the small sample size and number of patients lost to follow-up when contacted by telephone survey. As well, community clinics are 
within the vicinity of our hospitals and there is a possibility that these patients had some kind of incidental hospital contact in recent weeks (i.e. less than 12). A study performed on CA-CDI in a rural community where no hospitals are in the vicinity would rule this possibility out. Further limitations include data only acquired from a single centre (at least geographically); limited patient-level data available; observational study design; lack of inclusion of hospitalized patients with CA-CDI; lack of ability to distinguish $\mathrm{CDI}$ from $C$. difficile colonization; and recall or memory with a timespan of months between the occurrence of $\mathrm{CDI}$ and the telephone interview. Longitudinal studies may be of value where patients in the community are tested and followed over time in a prospective fashion. Nevertheless, there is a paucity of epidemiological and strain typing data on CA-CDI and these results support a community-nosocomial linkage even in cases traditionally attributed to the community.

\section{Abbreviations}

CA: Community acquired; CDI: Clostridium difficile infection;

DNA: Deoxyribonucleic acid; ECOFF: Epidemiological cut-off;

IBD: Inflammatory bowel disease; PFGE: Pulsed field gel electrophoresis;

WGS: Whole genome sequencing

\section{Acknowledgements}

We thank the medical laboratory technologists at Calgary Laboratory Services for their expert technical assistance. We thank Dr. Tarah Lynch for helpful discussion regarding bioinformatics analysis.

\section{Funding}

Funding for this work was provided in part by CIHR and Calgary Laboratory Services, Canada (DRP), Canadian Foundation for Infectious Diseases (CST), and research support from Abbott Laboratories, Inc. (CYC).

\section{Availability of data and materials}

Supporting data and materials can be obtained from the authors if within reason.

\section{Authors' contributions}

DRP and CYC designed the study. CST, GP, ALG and JER performed experiments. All authors contributed to the data analysis and writing. All authors have read and approved the manuscript.

\section{Ethics approval and consent to participate}

Ethical approval was obtained through University of Calgary Conjoint Health Research Ethics Board (ID 13-0406). Requirement for consent was waived.

\section{Consent for publication}

Not applicable.

\section{Competing interests}

DRP is on the BMC Editorial Board. The authors declare that they have no competing interests.

\section{Publisher's Note}

Springer Nature remains neutral with regard to jurisdictional claims in published maps and institutional affiliations.

\section{Author details}

'Department of Microbiology and Infectious Diseases, University of Calgary, Calgary, AB, Canada. 'Department of Medicine, University of Calgary, Calgary, $A B$, Canada. ${ }^{3}$ Calgary Laboratory Services, Calgary, AB, Canada. ${ }^{4}$ Department of Veterinary Microbiology, University of Saskatchewan, Regina, Canada. ${ }^{5}$ Department of Laboratory Medicine, University of California San Francisco,
San Francisco, California, USA. ${ }^{6}$ Department of Pathology and Laboratory Medicine, University of Calgary, Calgary, AB, Canada. 'Department of Laboratory Medicine, University of Washington, Seattle, WA, USA. ${ }^{8}$ Department of Medicine, Division of Infectious Diseases, University of California San Francisco, San Francisco, CA, USA. ${ }^{9}$ Diagnostic and Scientific Center, Room 1W-416, 9-3535 Research Road NW, Calgary, AB T2L 2K8, Canada.

Received: 17 April 2018 Accepted: 16 August 2018

Published online: 31 August 2018

\section{References}

1. Bartlett JG. Clinical practice. Antibiotic-associated diarrhea. N Engl J Med. 2002;346(5):334-9.

2. Miller BA, Chen LF, Sexton DJ, Anderson DJ. Comparison of the burdens of hospital-onset, healthcare facility-associated Clostridium difficile infection and of healthcare-associated infection due to methicillin-resistant Staphylococcus aureus in community hospitals. Infect Control Hosp Epidemiol. 2011;32(4):387-90.

3. Zilberberg MD, Shorr AF, Kollef MH. Growth and geographic variation in hospitalizations with resistant infections, United States, 2000-2005. Emerg Infect Dis. 2008:14(11):1756-8.

4. Beaugerie L, Flahault A, Barbut F, Atlan P, Lalande V, Cousin P, Cadilhac M, Petit JC, Study G. Antibiotic-associated diarrhoea and Clostridium difficile in the community. Aliment Pharmacol Ther. 2003;17(7):905-12.

5. Kandel C, Moayedi Y, Bunce PE. A woman with community-acquired Clostridium difficile infection. CMAJ. 2012;184(12):1383-5.

6. Lee L, Cohen SH. Community-aquired Clostridium difficle infection: an emerging problem. Curr Emerg Hosp Med Rep. 2013;1:149-53.

7. Bauer MP, Goorhuis A, Koster T, Numan-Ruberg SC, Hagen EC, Debast SB, Kuijper EJ, van Dissel JT. Community-onset Clostridium difficile-associated diarrhoea not associated with antibiotic usage--two case reports with review of the changing epidemiology of Clostridium difficile-associated diarrhoea. Neth J Med. 2008;66(5):207-11.

8. (CDC) CfDCaP. Severe Clostridium difficile-associated disease in populations previously at low risk--four states, 2005. MMWR Morb Mortal Wkly Rep. 2005; 54(47):1201-5.

9. (CDC) CfDCaP. Surveillance for community-associated Clostridium difficile-Connecticut, 2006. MMWR Morb Mortal Wkly Rep. 2006;57(13):340-3.

10. (CDC) CfDCaP. Vital signs: preventing Clostridium difficile infections. MMWR Morb Mortal Wkly Rep. 2012;61 (9):157-62.

11. McDonald LC, Coignard B, Dubberke E, Song X, Horan T, Kutty PK, Ad hoc Clostridium difficile surveillance working $\mathrm{G}$. Recommendations for surveillance of Clostridium difficile-associated disease. Infect Control Hosp Epidemiol. 2007;28(2):140-5.

12. Khanna S, Pardi DS, Aronson SL, Kammer PP, Orenstein R, St Sauver JL, Harmsen WS, Zinsmeister AR. The epidemiology of community-acquired Clostridium difficile infection: a population-based study. Am J Gastroenterol. 2012;107(1):89-95

13. Khanna S, Baddour LM, Huskins WC, Kammer PP, Faubion WA, Zinsmeister AR, Harmsen WS, Pardi DS. The epidemiology of Clostridium difficile infection in children: a population-based study. Clin Infect Dis. 2013:56(10):1401-6.

14. Wilcox MH, Mooney L, Bendall R, Settle CD, Fawley WN. A case-control study of community-associated Clostridium difficile infection. J Antimicrob Chemother. 2008:62(2):388-96.

15. Gupta A, Khanna S. Community-acquired Clostridium difficile infection: an increasing public health threat. Infect Drug Resist. 2014;7:63-72.

16. Sethi AK, Al-Nassir WN, Nerandzic MM, Bobulsky GS, Donskey CJ. Persistence of skin contamination and environmental shedding of Clostridium difficile during and after treatment of $C$. difficile infection. Infect Control Hosp Epidemiol. 2010;31(1):21-7.

17. Chitnis AS, Holzbauer SM, Belflower RM, Winston LG, Bamberg WM, Lyons $C$, Farley MM, Dumyati GK, Wilson LE, Beldavs ZG, et al. Epidemiology of community-associated Clostridium difficile infection, 2009 through 2011. JAMA Intern Med. 2013;173(14):1359-67.

18. Rodriguez-Palacios A, Reid-Smith RJ, Staempfli HR, Weese JS. Clostridium difficile survives minimal temperature recommended for cooking ground meats. Anaerobe. 2010;16(5):540-2.

19. Avbersek J, Janezic S, Pate M, Rupnik M, Zidaric V, Logar K, Vengust M, Zemljic M, Pirs T, Ocepek M. Diversity of Clostridium difficile in pigs and other animals in Slovenia. Anaerobe. 2009;15(6):252-5. 
20. Indra A, Lassnig H, Baliko N, Much P, Fiedler A, Huhulescu S, Allerberger F. Clostridium difficile: a new zoonotic agent? Wien Klin Wochenschr. 2009; 121(3-4):91-5.

21. Keel MK, Songer JG. The distribution and density of Clostridium difficile toxin receptors on the intestinal mucosa of neonatal pigs. Vet Pathol. 2007;44(6):814-22.

22. Pirs T, Ocepek M, Rupnik M. Isolation of Clostridium difficile from food animals in Slovenia. J Med Microbiol. 2008:57(Pt 6):790-2.

23. Weese JS, Avery BP, Rousseau J, Reid-Smith RJ. Detection and enumeration of Clostridium difficile spores in retail beef and pork. Appl Environ Microbiol. 2009;75(15):5009-11.

24. http://www.eucast.org/fileadmin/src/media/PDFs/EUCAST_files/Breakpoint_ tables/v_7.1_Breakpoint_Tables.pdf. [Accessed 17 April 2018].

25. Erikstrup LT, Danielsen TK, Hall V, Olsen KE, Kristensen B, Kahlmeter G, Fuursted K, Justesen US. Antimicrobial susceptibility testing of Clostridium difficile using EUCAST epidemiological cut-off values and disk diffusion correlates. Clin Microbiol Infect. 2012;18(8):E266-72.

26. Janezic S, Rupnik M. Molecular typing methods for Clostridium difficile: pulsed-field gel electrophoresis and PCR ribotyping. Methods Mol Biol. 2010;646:55-65.

27. Alfa MJ, Kabani A, Lyerly D, Moncrief S, Neville LM, Al-Barrak A, Harding GK, Dyck B, Olekson K, Embil JM. Characterization of a toxin A-negative, toxin B-positive strain of Clostridium difficile responsible for a nosocomial outbreak of Clostridium difficile-associated diarrhea. J Clin Microbiol. 2000;38(7):2706-14.

28. Klaassen $\mathrm{CH}$, van Haren HA, Horrevorts AM. Molecular fingerprinting of Clostridium difficile isolates: pulsed-field gel electrophoresis versus amplified fragment length polymorphism. J Clin Microbiol. 2002;40(1):101-4.

29. Tenover FC, Akerlund T, Gerding DN, Goering RV, Bostrom T, Jonsson AM, Wong E, Wortman AT, Persing DH. Comparison of strain typing results for Clostridium difficile isolates from North America. J Clin Microbiol. 2011:49(5):1831-7.

30. Khanna S, Pardi DS, Aronson SL, Kammer PP, Baddour LM. Outcomes in community-acquired Clostridium difficile infection. Aliment Pharmacol Ther. 2012;35(5):613-8.

31. Levy AR, Szabo SM, Lozano-Ortega G, Lloyd-Smith E, Leung V, Lawrence R, Romney MG. Incidence and costs of Clostridium difficile infections in Canada. Open Forum Infect Dis. 2015;2(3):ofv076.

32. Mori N, Aoki Y. Clinical characteristics and risk factors for communityacquired Clostridium difficile infection: a retrospective, case-control study in a tertiary care hospital in Japan. J Infect Chemother. 2015;21(12):864-7.

33. Kutty PK, Woods CW, Sena AC, Benoit SR, Naggie S, Frederick J, Evans S, Engel J, McDonald LC. Risk factors for and estimated incidence of community-associated Clostridium difficile infection, North Carolina, USA. Emerg Infect Dis. 2010;16(2):197-204.

34. Drummond LJ, McCoubrey J, Smith DG, Starr JM, Poxton IR. Changes in sensitivity patterns to selected antibiotics in Clostridium difficile in geriatric in-patients over an 18-month period. J Med Microbiol. 2003;52(Pt 3):259-63.

35. Brazier JS, Fawley W, Freeman J, Wilcox MH. Reduced susceptibility of Clostridium difficile to metronidazole. J Antimicrob Chemother. 2001;48(5):741-2.

36. Pelaez T, Alcala L, Alonso R, Rodriguez-Creixems M, Garcia-Lechuz JM, Bouza E. Reassessment of Clostridium difficile susceptibility to metronidazole and vancomycin. Antimicrob Agents Chemother. 2002;46(6):1647-50.

37. See I, Mu Y, Cohen J, Beldavs ZG, Winston LG, Dumyati G, Holzbauer S, Dunn J, Farley MM, Lyons C, et al. NAP1 strain type predicts outcomes from Clostridium difficile infection. Clin Infect Dis. 2014:58(10):1394-400.

38. Labbe AC, Poirier L, Maccannell D, Louie T, Savoie M, Beliveau C, Laverdiere $\mathrm{M}$, Pepin J. Clostridium difficile infections in a Canadian tertiary care hospital before and during a regional epidemic associated with the BI/NAP1/027 strain. Antimicrob Agents Chemother. 2008;52(9):3180-7.

39. Walker AS, Eyre DW, Wyllie DH, Dingle KE, Griffiths D, Shine B, Oakley S, O'Connor L, Finney J, Vaughan A, et al. Relationship between bacterial strain type, host biomarkers, and mortality in Clostridium difficile infection. Clin Infect Dis. 2013;56(11):1589-600.

40. Antharam VC, Li EC, Ishmael A, Sharma A, Mai V, Rand KH, Wang GP. Intestinal dysbiosis and depletion of butyrogenic bacteria in Clostridium difficile infection and nosocomial diarrhea. J Clin Microbiol. 2013;51(9):2884-92.

41. Chang JY, Antonopoulos DA, Kalra A, Tonelli A, Khalife WT, Schmidt TM, Young VB. Decreased diversity of the fecal microbiome in recurrent Clostridium difficile-associated diarrhea. J Infect Dis. 2008;197(3):435-8.

42. Seekatz AM, Young VB. Clostridium difficile and the microbiota. J Clin Invest. 2014;124(10):4182-9.

Ready to submit your research? Choose BMC and benefit from:

- fast, convenient online submission

- thorough peer review by experienced researchers in your field

- rapid publication on acceptance

- support for research data, including large and complex data types

- gold Open Access which fosters wider collaboration and increased citations

- maximum visibility for your research: over $100 \mathrm{M}$ website views per year

At BMC, research is always in progress.

Learn more biomedcentral.com/submissions 